\title{
ON THE CAUSAL PROPAGATION OF FIELDS
}

\author{
Göran Bergqvist \\ Department of Mathematics, Mälardalen University \\ 72123 Västerås, Sweden (gbt@mdh.se) \\ José M. M. Senovilla \\ Departamento de Física Teórica, Universidad del País Vasco \\ Apartado 644, 48080 Bilbao, Spain \\ and \\ Departament de Física Fonamental, Universitat de Barcelona \\ Diagonal 647, E-08028 Barcelona, Spain \\ (seno@ffn.ub.es)
}

August 15, 2018

\begin{abstract}
By using geometric methods and superenergy tensors, we find new simple criteria for the causal propagation of physical fields in spacetimes of any dimension. The method can be applied easily to many different theories and to arbitrary fields (such as scalar or electromagnetic ones). In particular, it provides a conservation theorem of the free gravitational field in all $N$-dimensional spacetimes conformally related to Einstein spaces (including the Ricci-flat case). In general relativity, our criteria provide simple proofs and a unified treatment of conservation theorems for neutrinos, photons, electrons and all other massless and massive free spin $n / 2$ fields. The uniqueness of the solution to the field equations also follows from our treatment under certain circumstances.
\end{abstract}

PACS: 04.20.Cv, 04.20.Ex, 03.65.Pm, 04.50.+h, 02.30.Jr 
The traditional way of studying general properties of field equations for physical fields on spacetimes is to introduce some coordinates and translate the geometric structure into the language of standard theory of partial differential equations to apply analytic methods or to consider the existence of fundamental solutions (propagators),

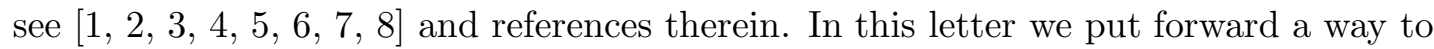
use the geometric information of tensorial field equations in a more direct manner. We show how a conservation theorem for fields can be proven, a theorem which provides information about the causal propagation of the fields. Our methods work for spacetimes of any dimension, so that it can be applied to many different theories including string theory, M-theory (see e.g. [9]), supergravity, gauge theories, Kaluza-Klein-like theories, Brans-Dicke and scalar-tensor theories, dilaton gravity and many others. The idea is to generalize the conservation theorem proved in [10] for the energy-momentum tensors that satisfy the dominant energy condition to the case of arbitrary physical fields. The key points in our reasoning are: first, "superenergy" tensors with the corresponding dominant property can always be constructed and, second, these tensors do not have to be related to any physically meaningful quantity, nor to the energymomentum tensor of the field (which, in fact, cannot be used in many cases, see below, including the important case of the gravitational field where it cannot be defined). The only condition in our conservation theorem is an assumption on the divergence of the superenergy tensor which is easy to check explicitly.

Our method is clearly related to the techniques of symmetric hyperbolic systems. Friedrich [1], 11] has used these techniques to study many interesting equations including several of the examples we will discuss here. The great advantage of our method is that we do not need to put the equations on a form which fits the theory of symmetric hyperbolic systems but we use the superenergy tensor directly in a typical integration procedure and to obtain estimates.

Let spacetime $V_{N}$ be an $N$-dimensional Lorentzian manifold with a metric $g$ of signature $(+,-, \ldots,-)$. A rank- $m$ tensor $T$ is said to satisfy the dominant property if 12, 13, 14

$$
T_{a_{1} \ldots a_{m}} u_{1}^{a_{1}} \ldots u_{m}^{a_{m}} \geq 0
$$

for any set $\left\{\vec{u}_{1}, \ldots, \vec{u}_{m}\right\}$ of causal future-pointing vectors. This is equivalent [14, 12] to that with respect to an orthonormal basis $\left\{\vec{e}_{0}, \ldots, \vec{e}_{N-1}\right\}$ the pure time component dominates any other component, that is

$$
T_{0 \ldots 0} \geq\left|T_{\alpha \ldots \beta}\right|
$$

for any $0 \leq \alpha, \ldots, \beta \leq N-1$. Note that this also implies that $T_{0 \ldots 0}=0 \Rightarrow T=$ 0 . (Observe that the dominant property is a generalization of the dominant energy condition for rank-2 energy-momentum tensors [10].) 
In general, a physical field $A$ will not have the dominant property but, as has been proven in complete generality [12, 13] and in arbitrary dimensions [14], one can always construct superenergy tensors $T(A)$ of $A$ which have the dominant property and such that, in any open neighbourhood,

$$
A=0 \Longleftrightarrow T(A)=0
$$

This property holds in open neighbourhoods of the manifold, but it may need a refinement at isolated points or lower-dimensional subsets. For instance, sometimes $T(A)$ vanishes at a point if and only if $A$ and its derivatives up to a certain order are zero [14], see for an explicit clear example the case of the massive scalar field below. We shall denote by $\mathcal{A}$ the set formed by the tensor $A$ and its derivatives up to the required order such that, at any point $x \in V_{N}$

$$
\left.\mathcal{A}\right|_{x}=\left.0 \Longleftrightarrow T(A)\right|_{x}=0 \text {. }
$$

The set $\mathcal{A}$ essentially contains the derivatives of $A$ appearing explicitly in the expression of $T(A)$ for each case.

The construction of $T(A)$ generalizes the definitions of energy-momentum tensors of scalar and Maxwell fields [12, 13, 14 and the traditional Bel and Bel-Robinson superenergy tensors of the gravitational field [12, 13, 14, 15, 16, 17]. Many examples of $T(A)$ will be given below. Note that the standard energy-momentum tensors of physical fields, even in the cases they are well defined, do not always have the dominant property 15] so they cannot be used as the tensor $T(A)$ in general for our purposes.

Let us try to see now under what conditions the physical field $A$ will propagate causally. To that end, assume that $S$ is an $(N-1)$-dimensional closed achronal set in $V_{N}$ and $D^{+}(S)$ its future Cauchy development (see [10] for notation and definitions). As $S$ is achronal, int $D^{+}(S)$ is globally hyperbolic and it can therefore be foliated by spacelike hypersurfaces $\Sigma_{t} \equiv\{t=$ const. $\}$, where the gradient $\boldsymbol{v}=d t$ is timelike everywhere in $\operatorname{int} D^{+}(S)$. Take any point $q \in D^{+}(S)$, then $K \equiv J^{-}(q) \cap \overline{D^{+}(S)}$ is compact and has boundary $\partial K=\tilde{S} \cup H^{+}(\tilde{S})$, where $\tilde{S} \equiv S \cap J^{-}(q)$ and $H^{+}(\tilde{S})$ is the future Cauchy horizon of $\tilde{S}$. Using any of the superenergy tensors $T(A)$ we define the superenergy integral

$w(t) \equiv \int_{J^{-}\left(\Sigma_{t}\right) \cap K} T^{a_{1} \ldots a_{m}}(A) v_{a_{1}} \ldots v_{a_{m}} \boldsymbol{\eta}=\int_{t_{0}}^{t}\left(\int_{\Sigma_{t^{\prime}}} T^{a_{1} \ldots a_{m}}(A) v_{a_{2}} \ldots v_{a_{m}} d \sigma_{a_{1}} \mid \Sigma_{t^{\prime}}\right) d t^{\prime}$

Here $J^{-}\left(\Sigma_{t}\right)$ denotes the causal past of $\Sigma_{t}, \boldsymbol{\eta}$ the canonical volume $N$-form on $V_{N}$, $\left.d \sigma_{a_{1}}\right|_{\Sigma_{t^{\prime}}}$ the volume $(N-1)$-form on $\Sigma_{t^{\prime}}$ pointing along $\vec{v}$, and $t_{0}$ the minimal value of $t$ in $K$. 
The procedure now follows the structure of a theorem for energy-momentum tensors in [10]. Differentiating, we get by Gauss' theorem

$$
\begin{gathered}
\frac{d w}{d t} \equiv w^{\prime}(t)=\left.\int_{\Sigma_{t}} T^{a_{1} \ldots a_{m}}(A) v_{a_{2}} \ldots v_{a_{m}} d \sigma_{a_{1}}\right|_{\Sigma_{t}}= \\
=-\left.\int_{H^{+}(\tilde{S}) \cap J^{-}\left(\Sigma_{t}\right)} T^{a_{1} \ldots a_{m}}(A) v_{a_{2}} \ldots v_{a_{m}} d \sigma_{a_{1}}\right|_{H^{+}(\tilde{S})}-\left.\int_{\tilde{S}} T^{a_{1} \ldots a_{m}}(A) v_{a_{2}} \ldots v_{a_{m}} d \sigma_{a_{1}}\right|_{\tilde{S}}+ \\
+\int_{J^{-}\left(\Sigma_{t}\right) \cap K}\left\{\left[\nabla_{a_{1}} T^{a_{1} \ldots a_{m}}(A)\right] v_{a_{2}} \ldots v_{a_{m}}+\right. \\
\left.+T^{a_{1} \ldots a_{m}}(A)\left[\left(\nabla_{a_{1}} v_{a_{2}}\right) v_{a_{3}} \ldots v_{a_{m}}+\ldots+v_{a_{2}} \ldots v_{a_{m-1}}\left(\nabla_{a_{1}} v_{a_{m}}\right)\right]\right\} \boldsymbol{\eta}
\end{gathered}
$$

where $\left.d \sigma_{a}\right|_{\zeta}$ always denotes the outward-pointing volume $(N-1)$-form on any $(N-1)$ dimensional subset $\zeta$. Given that $T(A)$ has the dominant property, by (4) and (5) we have

$$
w(t) \geq 0, \quad w^{\prime}(t) \geq 0 .
$$

By (11) we get that $T^{a_{1} \ldots a_{m}}(A)\left(\nabla_{a_{1}} v_{a_{2}}\right) v_{a_{3}} \ldots v_{a_{m}} \leq M_{2} T^{a_{1} \ldots a_{m}}(A) v_{a_{1}} \ldots v_{a_{m}}$ on $K$ for some constant $M_{2}$, because $\nabla_{a_{1}} v_{a_{2}}$ is bounded on the compact $K$. Similarily, we get constants $M_{3}, \ldots, M_{m}$ from the other terms so that putting $M \equiv M_{2}+\ldots+M_{m}$ and using the fact that the normal to $H^{+}(\tilde{S})$ pointing outwards from $K$ is future-pointing, we get by $(6)$ that

$0 \leq w^{\prime}(t) \leq M w(t)-\left.\int_{\tilde{S}} T^{a_{1} \ldots a_{m}}(A) v_{a_{2}} \ldots v_{a_{m}} d \sigma_{a_{1}}\right|_{\tilde{S}}+\int_{J^{-}\left(\Sigma_{t}\right) \cap K}\left[\nabla_{a_{1}} T^{a_{1} \ldots a_{m}}(A)\right] v_{a_{2}} \ldots v_{a_{m}} \boldsymbol{\eta}$.

If $\mathcal{A}=0$ on $\tilde{S}$ (we usually think of $S$ as an initial slice), then due to the property (3) of the superenergy tensors $T(A)$ we obtain

$$
0 \leq w^{\prime}(t) \leq M w(t)+\int_{J^{-}\left(\Sigma_{t}\right) \cap K}\left[\nabla_{a_{1}} T^{a_{1} \ldots a_{m}}(A)\right] v_{a_{2}} \ldots v_{a_{m}} \boldsymbol{\eta} .
$$

Consider now the following divergence condition 円:

$$
\nabla_{a_{1}} T^{a_{1} \ldots a_{m}}(A) v_{a_{2}} \ldots v_{a_{m}} \leq h T^{a_{1} \ldots a_{m}}(A) v_{a_{1}} \ldots v_{a_{m}}
$$

where $h$ is a continuous function on $\overline{D^{+}(S)}$. Then eq. (77) becomes simply

$$
0 \leq w^{\prime}(t) \leq(M+H) w(t)
$$

\footnotetext{
${ }^{1}$ This is enough for our purposes, but it can be obviously generalized to other more general conditions involving "powers" of the tensor $T$ greater than one. On the other hand, (8) can be written in a form explicitly independent of the foliation, but this is not needed here.
} 
where the constant $H$ is the maximum of $h$ on $K$. Integrating this relation (or using a rather simple Gronwall's inequality) and using $w\left(t_{0}\right)=0$, one then arrives at

$$
w(t) \equiv 0 .
$$

This means that $T_{0 \ldots 0}(A) \equiv 0$ on $K$ (with $\vec{e}_{0} \propto \vec{v}$ ) and hence $T(A) \equiv 0$ on $K$. For any point $q \in D^{+}(S)$ we can apply the above reasoning to the corresponding compact $K$ to obtain $T(A)=0$ on $K$ and hence we get that $T(A)=0$ on $\overline{D^{+}(S)}$ by continuity, which is equivalent to $A=0$ on $K$ due to (2). Note that changing future to past we also get $A=0$ in $\overline{D^{-}(S)}$ and hence in $\overline{D(S)}=\overline{D^{+}(S) \cup D^{-}(S)}$.

Using the terminology of [10], we have thus proven the following

Conservation theorem: $\left.\mathcal{A}\right|_{S}=\left.0 \Longrightarrow A\right|_{\overline{D(S)}}=0$ if the superenergy tensor $T(A)$ satisfies (8).

This theorem is clearly related to the causal propagation of the field described by $A$, because if $A \neq 0$ in any region arbitrarily close to $\overline{D(S)}$ then $A$ will propagate in time according to the field equations but it will never be able to "enter" $\overline{D(S)}$, showing that $A$ cannot travel faster than light. On the other hand, the universality of the superenergy construction [12, 13, 14] is essential here.

As an example in arbitrary dimension, consider a scalar field $\phi$ with mass $m$ satisfying the 'generalized Klein-Gordon' $N$-dimensional equation $\left(\square+m^{2}\right) \phi=k^{a} \nabla_{a} \phi$, where $k^{a}$ is some continuous vector field. Define the tensor $T(\phi)$ as

$$
T_{a b}=\nabla_{a} \phi \nabla_{b} \phi-\frac{1}{2} g_{a b} \nabla_{c} \phi \nabla^{c} \phi+\frac{1}{2} m^{2} \phi^{2} g_{a b}
$$

(this is the standard energy-momentum tensor if $k^{a}=0$, in which case $T(\phi)$ is divergence-free). $T(\phi)$ is easily shown to have the dominant property in any dimension and to be zero if and only if $\phi=0$ (iff $\nabla_{a} \phi=0$ for the massless case) in open neighbourhoods [14]. However, at points $x \in V_{N}$, we have [14]

$$
\begin{aligned}
\left.T(\phi)\right|_{x}=0 & \left.\Longleftrightarrow \phi\right|_{x}=0,\left.\nabla \phi\right|_{x}=0 & \text { if } m \neq 0 \\
\left.T(\phi)\right|_{x}=0 & \left.\Longleftrightarrow \nabla \phi\right|_{x}=0 & \text { if } m=0
\end{aligned}
$$

Thus, in this case the set $\mathcal{A}$ is given by $\mathcal{A} \equiv\{\phi, \nabla \phi\}$ in the massive case and by $\mathcal{A} \equiv \nabla \phi$ in the massless one. In any case, $T(\phi)$ satisfies the divergence condition (8) so that the theorem shows that $\left.\phi\right|_{S},\left.\nabla \phi\right|_{S}=\left.0 \Longrightarrow \phi\right|_{\overline{D(S)}}=0$ if $m \neq 0$, $\left(\left.\nabla \phi\right|_{S}=\left.0 \Longrightarrow \phi\right|_{\overline{D(S)}}=\right.$ const. if $\left.m=0\right)$. In addition, due to the linearity of the generalized Klein-Gordon equation, this result implies that $\left.\phi\right|_{\overline{D(S)}}$ is unique given the initial condition $\left.\phi\right|_{S},\left.\nabla \phi\right|_{S}$. A similar proof works for the electromagnetic field using the energy-momentum tensor in dimension $N$. 
Much more interesting is the case of the free gravitational field in $N$ dimensions, because then no energy-momentum tensor exists (due to the Equivalence Principle and the non-localizability of the gravitational energy). However, one can use the superenergy tensors as follows. Let $\mathcal{T}$ be the generalized Bel-Robinson tensor given by 114

$$
\begin{gathered}
\mathcal{T}_{a b c d}=C_{a}{ }^{e}{ }_{c}^{f} C_{b e d f}+C_{a}{ }^{e}{ }_{d}^{f} C_{b e c f}-\frac{1}{2} g_{a b} C^{l e}{ }_{c}{ }^{f} C_{\text {ledf }}-\frac{1}{2} g_{c d} C_{a}{ }^{\text {elf }} C_{\text {belf }}+\frac{1}{8} g_{a b} g_{c d} C^{\text {helf }} C_{\text {helf }}, \\
\mathcal{T}_{a b c d}=\mathcal{T}_{b a c d}=\mathcal{T}_{a b d c}=\mathcal{T}_{c d a b} \\
\mathcal{T}^{a}{ }_{a c d}=(N-4) \mathcal{T}^{a}{ }_{c a d}, \quad \mathcal{T}^{a}{ }_{c a d}=-\frac{1}{2}\left(C_{\text {clef }} C_{d}{ }^{\text {lef }}-\frac{1}{4} g_{c d} C^{\text {helf }} C_{\text {helf }}\right)
\end{gathered}
$$

where $C$ is the Weyl tensor. Then $\mathcal{T}$ has the dominant property [14] (see [15, 16, 17] for $N=4$, in which case $\mathcal{T}$ is completely symmetric and traceless) and vanishes at any point if and only if $C$ is zero there [14]. Let $\tilde{g}$ be any metric conformally related to $g$ by

$$
\tilde{g}=e^{2 U} g
$$

Then $\tilde{C}^{a}{ }_{b c d}=C^{a}{ }_{b c d}$ and

$$
\tilde{\nabla}_{a} \tilde{C}_{b c d}^{a}=\nabla_{a} C_{b c d}^{a}+(N-3) U_{, a} C^{a}{ }_{b c d}
$$

which implies

$$
\begin{aligned}
\tilde{\nabla}_{a} \tilde{\mathcal{T}}^{a}{ }_{b c d}=e^{-2 U}\left[\nabla_{a} \mathcal{T}^{a}{ }_{b c d}\right. & +(N-4) U_{, a} \mathcal{T}^{a}{ }_{b c d}+U_{, a}\left(\mathcal{T}^{a}{ }_{c b d}+\mathcal{T}^{a}{ }_{d b c}\right)- \\
& \left.-(N-4) U_{, b} \mathcal{T}^{a}{ }_{c a d}-U_{, c} \mathcal{T}^{a}{ }_{b a d}-U_{, d} \mathcal{T}^{a}{ }_{b a c}\right] .
\end{aligned}
$$

If $\nabla_{a} C^{a} b c d=0$, then $\nabla_{a} \mathcal{T}^{a} b c d=0$ and by (12) we see that the divergence condition holds for $\tilde{\mathcal{T}}$. This comprises all spacetimes conformally related to Einstein spaces $\left(R_{a b}=\Lambda g_{a b}\right)$, including the important case of Ricci-flat metrics. Thus we have a simple proof of the causal propagation of free gravity (the Weyl tensor) in all such spacetimes, which are of big generality. The special case of General Relativity (where $N=4$ and the Bel-Robinson tensor is divergence-free) was studied in [16.

In the case of General Relativity, for many fields one can construct a tensor $T$ satisfying (8). Note that the energy-momentum tensors, which would be obvious candidates, are not always well defined (as happens for massless fields of spin higher than one), and that in many cases, such as for instance spin-1/2 fields, the standard energymomentum tensors do not satisfy the dominant energy condition 15]. Therefore, we must construct some other tensor $T$ in these cases in the same way as with the generalized Bel-Robinson tensor above. As mentioned before, in fact one can always construct a superenergy tensor $T(A)$ of any field $A$ with the dominant property [12, 13, 14]. 
Consider a massless spin $n / 2$ field $\varphi$. This is described by a symmetric spinor field $\varphi_{A_{1} \ldots A_{n}}=\varphi_{\left(A_{1} \ldots A_{n}\right)}$ (spinor indices are capital letters; see 15 for notation) and the field equation is 15$]$

$$
\nabla^{A_{1} B^{\prime}} \varphi_{A_{1} \ldots A_{n}}=0 .
$$

For the neutrino $(n=1)$ and the photon $(n=2)$ this is a well-posed initial value problem, but for $n>2$ there arise the Buchdahl algebraic constraints [18] involving the curvature for existence of solutions. We refer to [18, 15] for a discussion on these restrictions. Nonetheless, independetly of these restrictions, we can apply our method to prove causal propagation of the solutions in general.

Define the tensor $T(\varphi)$ by

$$
T_{a_{1} \ldots a_{n}}=\varphi_{A_{1} \ldots A_{n}} \bar{\varphi}_{A_{1}^{\prime} \ldots A_{n}^{\prime}}
$$

(with the usual identification $a_{1} \leftrightarrow A_{1} A_{1}^{\prime}$ between tensor and spinor indices [15] and where the bar denotes complex conjugation). As shown in [13], any sum of spinors squared in this sense has the dominant property. Note that for $n=2$ this is the usual energy-momentum for Maxwell fields. By (13), $\nabla^{a_{1}} T_{a_{1} \ldots a_{n}}=0$ and as $\left.T(\varphi)\right|_{x}=0 \Leftrightarrow$ $\left.\varphi\right|_{x}=0$, the conservation theorem is valid for $\varphi$. The theorem also holds for more general field equations. In analogy with the generalized Klein-Gordon $N$-dimensional equation, we can consider the simple generalization

$$
\nabla^{A_{1} B^{\prime}} \varphi_{A_{1} \ldots A_{n}}=k^{A_{1} B^{\prime}} \varphi_{A_{1} \ldots A_{n}}
$$

for some continuous vector field $k^{a}$. Then the divergence condition is also satisfied and therefore the theorem is applicable to such fields too. In any case, the uniqueness of $\varphi$ on $\overline{D(S)}$ for a given initial condition $\left.\varphi\right|_{S}$ holds.

For massive spin $n / 2$ fields, one can find consistent systems of equations (without any Buchdahl's constraints) for spinor fields $\varphi, \chi$ such as [19], 20]

$$
\nabla^{A_{1}}{ }_{B^{\prime}} \varphi_{A_{1} \ldots A_{n}}=\mu \chi_{A_{2} \ldots A_{n} B^{\prime}}, \quad \nabla_{\left(A_{1}\right.}{ }^{B^{\prime}} \chi_{\left.A_{2} \ldots A_{n}\right) B^{\prime}}=\nu \varphi_{A_{1} \ldots A_{n}}
$$

with $\mu, \nu \neq 0$ and $m^{2}=2 \mu \nu$. This is a well posed initial value problem for all spins. Let

$$
T_{a_{1} \ldots a_{n}}=\alpha \varphi_{A_{1} \ldots A_{n}} \bar{\varphi}_{A_{1}^{\prime} A_{2}^{\prime} \ldots A_{n}^{\prime}}+\beta \chi_{A_{1}^{\prime} A_{2} \ldots A_{n}} \bar{\chi}_{A_{1} A_{2}^{\prime} \ldots A_{n}^{\prime}}
$$

with constants $\alpha, \beta>0$. Then $T$ has the dominant property, $\left.T\right|_{x}=\left.0 \Leftrightarrow \varphi\right|_{x}=0=\left.\chi\right|_{x}$ and

$$
\begin{gathered}
\nabla^{a_{1}} T_{a_{1} \ldots a_{n}}=(\beta \nu-\alpha \mu) \chi_{A_{1}^{\prime} A_{2} \ldots A_{n}} \bar{\varphi}^{A_{1}^{\prime}} A_{2}^{\prime} \ldots A_{n}^{\prime}+ \\
+\frac{\beta n(n-1)}{\mu(n+1)} \epsilon^{A_{1}}{ }_{\left(A_{2}\right.} \Psi_{A_{3}}{ }^{D E F} \varphi_{\left.A_{4} \ldots A_{n}\right) D E F} \bar{\chi}_{A_{1} A_{2}^{\prime} \ldots A_{n}^{\prime}}+\text { complex conjugates. }
\end{gathered}
$$


Here we have used eq.(C1) of [20] and $\Psi$ denotes the Weyl spinor. For any $\alpha$ and $\beta$, any product of two components of $\chi$ and $\varphi$ with a bounded coefficient will be smaller than a constant times the time component $T_{0 \ldots 0}$ of $T$ (so it is not necessary to make the natural choice $\beta \nu=\alpha \mu)$. Thus the divergence condition (8) is satisfied and $(\varphi, \chi)$ propagate causally. The uniqueness of $(\varphi, \chi)$ on $\overline{D(S)}$ given the initial condition $\left.(\varphi, \chi)\right|_{S}$ follows again.

It is important to remark that the fact that $\mu$ is a constant is fundamental in the above reasoning, because it allows to isolate the derivative of $\chi$ (eq.(C1) in 20]) which in turn is used to check the divergence condition. Thus, for instance, the case of a massless spin-3/2 field satisfies the Rarita-Schwinger equations which, due to the Buchdahl constraints mentioned before, must be modified in curved spacetime in an appropriate manner, see e.g. [15, 21]. The resulting equations in Ricci-flat spacetimes are formally similar to (14) with $n=3$, but instead of the constant $\mu$ there appears the Weyl spinor $\Psi$ contracted appropriately with $\chi$ [21]. And this makes a big difference, for the derivative of $\chi$ cannot be isolated now in general (it requires an invertibility condition on the Weyl spinor). Then, if we construct the corresponding superenergy tensor (15), which still satisfies the dominant and other required properties, it is impossible to check the divergence condition generically. This is in accordance with the well-known problems of the causal propagation of Rarita-Schwinger fields in curved backgrounds (see e.g. [22]), which are reminiscent of the classical Velo-Zwanziger causal paradox 23] appearing when coupled to electromagnetic and other external fields due again to the existence of some invertibility conditions, see e.g. 24].

To summarize, we have presented an extremely simple and fully geometric argument for the causal propagation of physical fields in arbitrary dimensions. A sufficient condition, in many cases very easy to check explicitly, for causal propagation is given in terms of the divergence of an adequate superenergy tensor of the field. All our treatment is classical, although it may be also useful in quantum aspects of the referred theories. In this sense, notice that if any field may be described by tachyonic particles then the divergence condition, which is related to the field equations, must be violated. In general, we can choose to use any $T(A)$ with property (3) that satisfies the divergence condition if this is helpful, and in fact there usually exists an $s$-parameter family of such tensors for a natural number $s$ [14]. Nevertheless, from (舟) it is clear that only the symmetric part of $T(A)$ matters and thus the divergence condition refers to this intrinsically unique superenergy tensor (see also the discussion in 14]). On the other hand, sometimes even the completely symmetric tensors will not be unique (for instance in the case of (15), where $\alpha$ and $\beta$ remain arbitrary), but in these cases the divergence condition will hold for all of them and the conclusion about causal propagation will of course be definitive. Furthermore, the condition (8) can be weakened, for it does not need to hold pointwise but only on integrated form (even weaker conditions 
on the integral in (7) can actually be imposed for the conclusion (9) to follow). One can also formulate the conservation theorem for sets more general than $\overline{D(S)}$, but we have restricted ourselves to a physically interesting situation.

We believe that more sophisticated theorems for physical fields can be found using superenergy tensors to define norms and to do a priori estimates (as in [25]; with respect to this, see also the excellent and very recent review [26]). The fact that it is always possible to construct a superenergy tensor with the dominant property of any field is fundamental here. Closer connections with the theory of symmetric hyperbolic systems need to be investigated because it may well happen that our divergence condition (which is obviously connected to the field equations for $A$ ) provide some useful criteria for the hyperbolic symmetrizability of some field equations. All in all, we wish to stress the simplicity of our argument and the universality of its application.

G.B. is grateful to The Swedish Natural Science Research Council (NFR) for financial support.

\section{References}

[1] R.Courant and D.Hilbert, Methods of mathematical physics, Vol.II: Partial differential equations, Interscience, New York, 1962.

[2] F.G.Friedlander The wave equation on a curved spacetime, Cambridge University Press, Cambridge 1975

[3] Y.Choquet-Bruhat and J.W.York, in General Relativity and Gravitation, Vol. 1, ed. A.Held, Plenum, New York 1980

[4] H.Friedrich, Class.Quantum Grav.13, 1451, 1996

[5] A.Anderson, Y.Choquet-Bruhat, J.W.York, Topol.Meth.Nonlinear Anal. 10, 353, 1997

[6] O.Reula, Living Reviews, http://www.livingreviews.org/Articles/Volume1/1998-3reula

[7] A.Rendall, Living Reviews, http://www.livingreviews.org/Articles/Volume1/1998-4rendall

[8] M.Á.G.Bonilla, Class.Quantum Grav.15, 2001, 1998

[9] G.W.Gibbons, in Gravitation and Relativity: At the turn of the Millenium, (Proc. of the GR-15 Conference), eds. N.Dadhich and J.Narlikar, IUCAA, Pune, India, 1998

[10] S.W.Hawking and G.F.R.Ellis, The large-scale structure of spacetime, Cambridge University Press, Cambridge 1973

[11] H.Friedrich, J.Diff.Geom.17, 125, 1991

[12] J.M.M.Senovilla, to appear in Gravitation and Relativity in General, eds A.Molina, J.Martín, E.Ruiz and F.Atrio, World Scientific, Singapore 1999 (gr-qc/9901019) 
[13] G.Bergqvist, Mälardalen University preprint, 1999, to appear in Commun.Math.Phys.

[14] J.M.M.Senovilla, submitted (gr-qc/9906087).

[15] R.Penrose and W.Rindler, Spinors and spacetime, vol.1-2, Cambridge University Press, Cambridge 1986

[16] M.Á.G.Bonilla and J.M.M.Senovilla, Phys.Rev.Lett. 78, 783, 1997

[17] G.Bergqvist, J.Math.Phys.39, 2141, 1998

[18] H.A.Buchdahl, Nuovo Cim. 10, 96, 1958; 25, 485, 1962

[19] H.A.Buchdahl, J.Phys.A: Math.Gen.15, 1, 1982; 1057, 1982

[20] R.Illge, Class.Quantum Grav.13, 1499, 1996

[21] G. Silva-Ortigosa, Gen. Rel. Grav.30, 45, 1998

[22] G.R. Allcock and S.F. Hall, J.Phys.A: Math.Gen.11, 2305, 1978

[23] G. Velo and D. Zwanziger, Phys. Rev.186, 1337, 1969

[24] M. Kobayashi and Y. Takahashi, Prog. Theor. Phys.75, 993, 1986; J.Phys.A: Math.Gen.20, 6581, 1987; Nuovo. Cim. B104, 67, 1989

[25] D.Christodoulou and S.Klainerman, The global non-linear stability of Minkowski space, Princeton University Press, Princeton 1993

[26] S. Klainerman and F. Nicolò, Class. Quantum Grav. 16, R73, 1999. 